\title{
Diagnosis of primary hyperventilation syndrome by polysomnography: A report of two cases
}

\author{
TARANG N SHETH BSc, STEVEN KESTEN MD \\ The Toronto Hospital, University of Toronto, Toronto, Ontario
}

\begin{abstract}
TN SHETH, S KESTEN. Diagnosis of primary hyperventilation syndrome by polysomnography: A report of two cases. Can Respir J 1996;3(3):199-201.

Two cases of primary hyperventilation syndrome misdiagnosed as asthma are reported. Polysomnography was used to diagnose the syndrome and to confirm the psychogenic etiology of the patients' symptoms.
\end{abstract}

Key Words: Polysomnography, Primary hyperventilation syndrome

$\mathrm{P}$ rimary hyperventilation syndrome is diagnosed when alveolar ventilation is chronically increased with no identifiable organic or metabolic cause (1). The syndrome is characterized by dyspnea, presyncope, diaphoresis, palpitations and paraesthesias (2). Because dyspnea is often the major presenting symptom, the diagnosis can be confused with that of asthma.

While polysomnography is used primarily in the diagnosis of sleep disordered breathing, it will also document respiratory rate and tidal volume when voluntary control is removed (ie, sleep). In the two patients described in the pres-

\section{Diagnostic d'un syndrome d'hyperventilation primaire par polysomnographie: Rapport de deux cas}

RÉSUMÉ : Deux cas de syndrome d'hyperventilation primaire diagnostiqués à tort comme asthme sont rapportés. On a utilisé la polysomnographie pour diagnostiquer le syndrome et pour confirmer l'étiologie psychogène des symptômes des patients.

Correspondence: Dr Steven Kesten, Rush-Presbyterian St Luke's Medical Center, 1725 W Harrison Street, Suite 836, Chicago, IL 60612, USA. Telephone 312-563-2251, fax 312-563-2020 


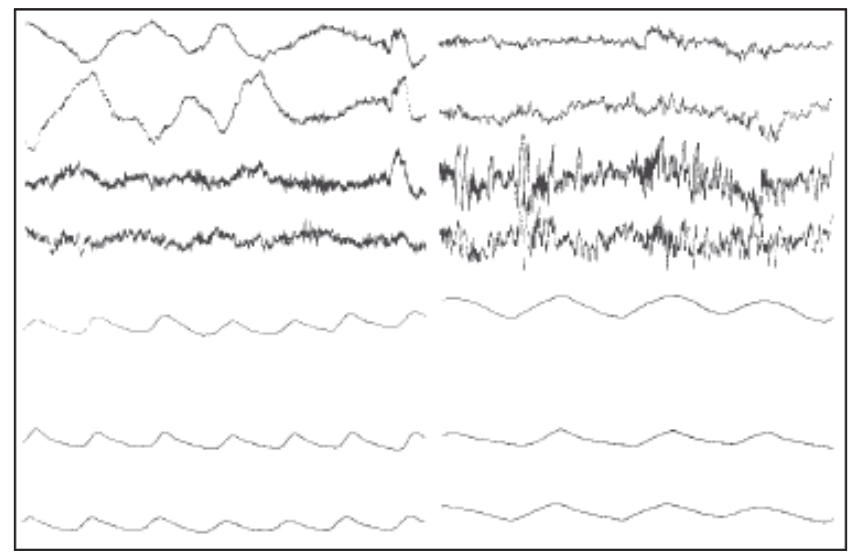

Figure 1) Recordings from electrooculogram (lines 1 and 2), electroencephalogram (lines 3 and 4) and respiratory inductance plethysmography (rig cage, abdomen and sum of both; lines 4 to 6) during resting wakefulness (left) and during stage 2 sleep (right). Each section is a 15 s recording

time was treated with nebulized bronchodilators and often with augmented systemic steroids. Despite intensive treatment for asthma, the patient's dyspnea was not well controlled and had gradually worsened to the point where she was dyspneic at rest and was limited to walking approximately 300 feet.

On multiple examinations, respiratory rate was between 20 and 30 breaths/min and noted to be shallow. Blood pressure and pulse were normal. An expiratory wheeze, most prominent over the trachea, was noted on multiple occasions. Cardiovascular examination was normal. Spirometry was not reproducible, and it appeared that the patient did not consistently inspire to total lung capacity; however, carbon monoxide uptake was normal. Chest radiographs were normal. Arterial blood gases drawn on room air in August 1994 were as follows: $\mathrm{pH} 7.48, \mathrm{PaCO}_{2} 27 \mathrm{mmHg}, \mathrm{PaO}_{2} 84 \mathrm{mmHg}$, bicarbonate $20 \mathrm{mmol} / \mathrm{L}$ and oxygen saturation $97 \%$. Bronchoscopy showed normal vocal cord movement. Fluoroscopy of the diaphragm and a two-dimensional echocardiogram were normal. A hyperventilation syndrome was suspected.

Polysomnography documented sleep architecture marked by a moderately severe alpha-electroencephalographic (EEG) arousal disturbance and frequent stage changes (18/h). Respiratory recordings showed an apnea/hypopnea index of 10.2 events/h, comprising 31 central apneas of 14 to $34 \mathrm{~s}$ duration and 13 hypopneas. Sleep onset central apneas were also noted and were associated with prolonged intervals of intervening wakefulness during which 2 to 5 min episodes of hyperventilation were observed (Figure 1). Arterial oxygen saturation remained between $94 \%$ and $98 \%$ throughout the night.

The patient was admitted to the respirology service in an attempt to withdraw her asthma medication and to administer a trial of benzodiazepine therapy. A psychiatric consultation suggested the presence of an anxiety disorder. The patient became resentful of the psychiatric consultation and refused anxiolytics and further psychiatric follow-up. She continued to use her asthma medications after her discharge.

\section{CASE 2}

A 37-year-old woman was diagnosed with 'childhood asthma' at the age of 16 years. Her symptoms, worsened by exercise, cold air and smoking, were initially well controlled with salbutamol and occasional low doses of beclomethasone. The patient had a 15 pack-year smoking history but quit in 1989 after she developed a severe, acute attack of dyspnea requiring hospital admission. Her exercise intolerance progressively worsened to the point where she was severely limited in function and was unable to walk one block without symptoms.

Physical examination revealed a rapid and shallow breathing pattern. Respiratory rate at rest was approximately $30 / \mathrm{min}$. Blood pressure was $170 / 110 \mathrm{mmHg}$, and heart rate was 104 beats/min and regular. The chest excursion was decreased. No wheezing was evident. There was a question of an upper airway grunt. Decreased breath sounds were noted bilaterally, although the patient's cooperation was questionable. Cardiovascular examination was normal. The patient was unable to perform reproducible spirometry. Chest radiograph was normal. Arterial blood gases drawn on room air were $\mathrm{pH} 7.42, \mathrm{PaCO}_{2} 35 \mathrm{mmHg}, \mathrm{PaO}_{2} 72 \mathrm{mmHg}$, bicarbonate $23 \mathrm{mmol} / \mathrm{L}$ and oxygen saturation 95\%. Phrenic and peripheral nerve stimulation were normal.

Polysomnography documented sleep architecture marked by frequent sleep stage changes $(26 / \mathrm{h})$, a severe alpha-EEG arousal disturbance, often associated with movement arousals $(13 / \mathrm{h})$ and leg movements. Respiratory recordings revealed an apnea/hypopnea index of 3.2, comprising one central and three obstructive apneas of 10 to $15 \mathrm{~s}$ duration. Of note were several episodes of erratic shallow breathing occurring exclusively during periods of intervening wakefulness. Arterial oxygen saturation remained well preserved, between $92 \%$ and $97 \%$ at all times.

After the sleep study it was felt that the patient might benefit from psychotherapy to address the role of anxiety in the onset of her symptoms. A psychiatric consultation was arranged, but although she attended one session, she failed to appear for further therapy.

\section{DISCUSSION}

A study in 1950 suggested that primary, chronic hyperventilation syndrome accounted for up to $10 \%$ of visits to general internists (1). However, further documentation of the incidence of this disorder is lacking. Despite its proposed relatively high incidence, considerable confusion exists in clinical practice about its precise definition and diagnosis (2). Because the presenting symptom is often persistent dyspnea, as in both cases documented above, hyperventilation syndrome can be confused with asthma. In addition, changes associated with the syndrome may produce bronchoconstriction, contributing to the sensation of dyspnea and simulating asthma (3), as may have been the case in our first patient. Misdiagnosis in both cases resulted in prolonged inappropriate use of asthma medications, including systemic corticosteroids and regular inhaled steroids with the concomitant unnecessary exposure to potential side effects. 
A careful evaluation of the physical findings in our two cases suggested that asthma and cardiac disease were unlikely. A rapid, shallow breathing pattern was uncharacteristic of asthma, as was a very variable spirometry performance. As well, there were no physical findings to suggest pulmonary hypertension. Chest radiographs were normal in both patients; two-dimensional echocardiography was normal in one.

Tools that are useful in assisting in the diagnosis include hyperventilation provocation tests and capnography (4). Our experience with the present patients suggests that polysomnography may be diagnostic of primary hyperventilation syndrome in the appropriate clinical scenario. In our patients the sleep study results indicated a direct association between the presence of hyperventilation and wakefulness. In addition, the pattern of hyperventilation cessation immediately upon sleep and immediate return during periods of wakefulness strongly suggested a psychogenic cause for this syndrome in both of our patients. Central apneas were probably secondary to the metabolic alkalosis induced by hyperventilation.

Reviews and commentaries regarding hyperventilation syndrome in the literature suggest an important role for anxiety or panic disorders in disease etiology $(2,5)$. It has been reported that perhaps more than $50 \%$ of patients with primary hyperventilation syndrome may have a coexisting panic dis- order and it may be that these two conditions are closely linked, sharing a hypersensitivity of a central alarm system (6). If this is indeed the case, then polysomnography may prove very useful in the diagnosis as it documents breathing patterns when the patient is awake and thus in a susceptible state and when the patient is asleep, thereby eliminating awareness and voluntary control. Clear differences between the two states, as in our patients, confirm the presence of primary hyperventilation syndrome and point to a possible psychogenic etiology.

The diagnosis of primary hyperventilation syndrome thus poses an interesting problem for clinicians, in which polysomnography may have an important role to play when diagnostic uncertainty exists.

\section{REFERENCES}

1. Rice R. Symptom patterns of the hyperventilation syndrome. Am J Med Sci 1950;8:691-700.

2. Howell JBL. Behavioural breathlessness. Thorax 1990;45:287-92

3. Ferguson A, Addington W, Gaensler E. Dyspnea and bronchospasm from inappropriate postexercise hyperventilation. Ann Intern Med 1969;71:1063-72.

4. Vansteenkiste J, Rochette F, Demedts M. Diagnostic tests of hyperventilation syndrome. Eur Respir J 1991:4;393-9.

5. Pfeffer JM. Hyperventilation and the hyperventilation syndrome. Postgrad Med J 1984;60(Suppl 2):12-5.

6. Cowley DS, Roy-Byrne PP. Hyperventilation and panic disorder. Am J Med 1987;83:929-37. 


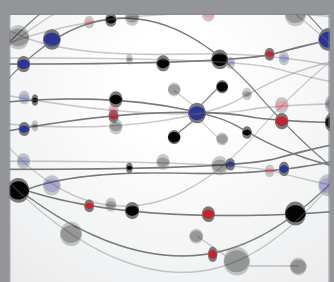

The Scientific World Journal
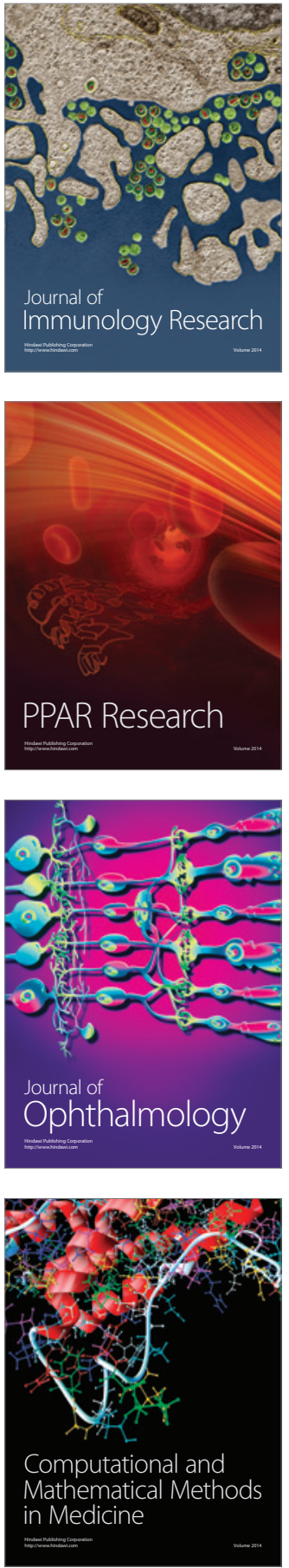

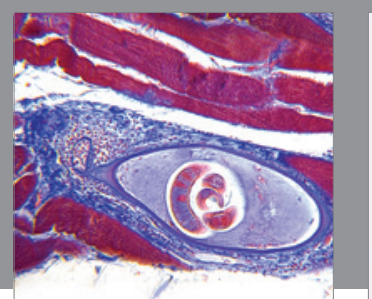

Gastroenterology Research and Practice

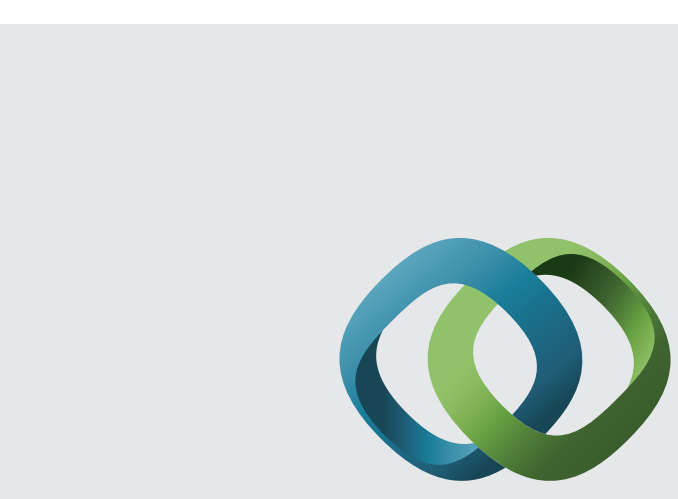

\section{Hindawi}

Submit your manuscripts at

http://www.hindawi.com
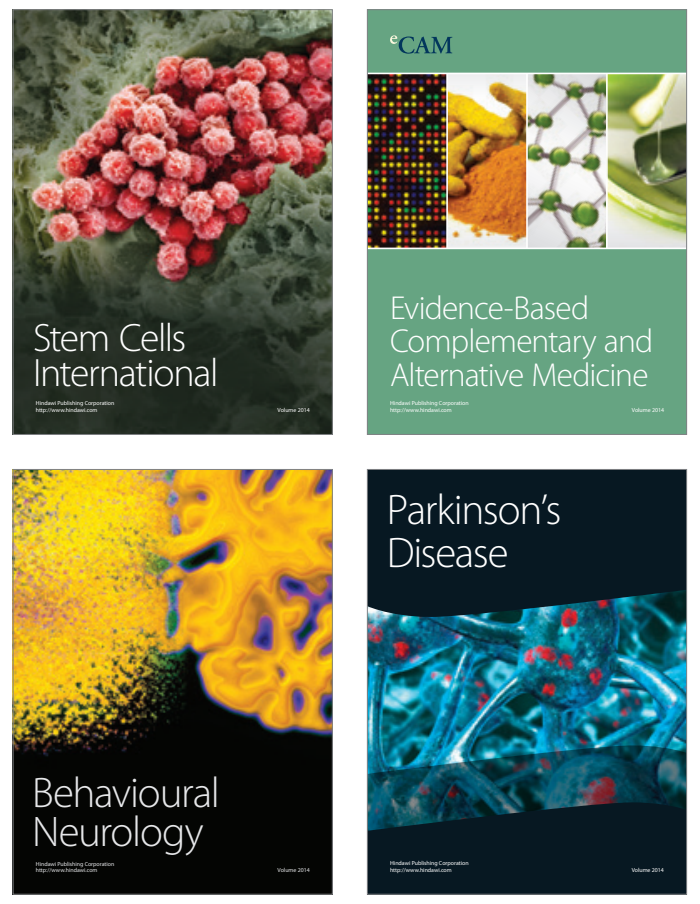
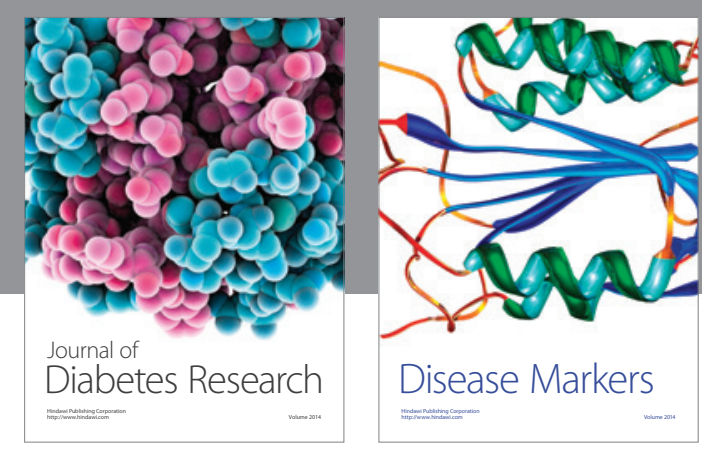

Disease Markers
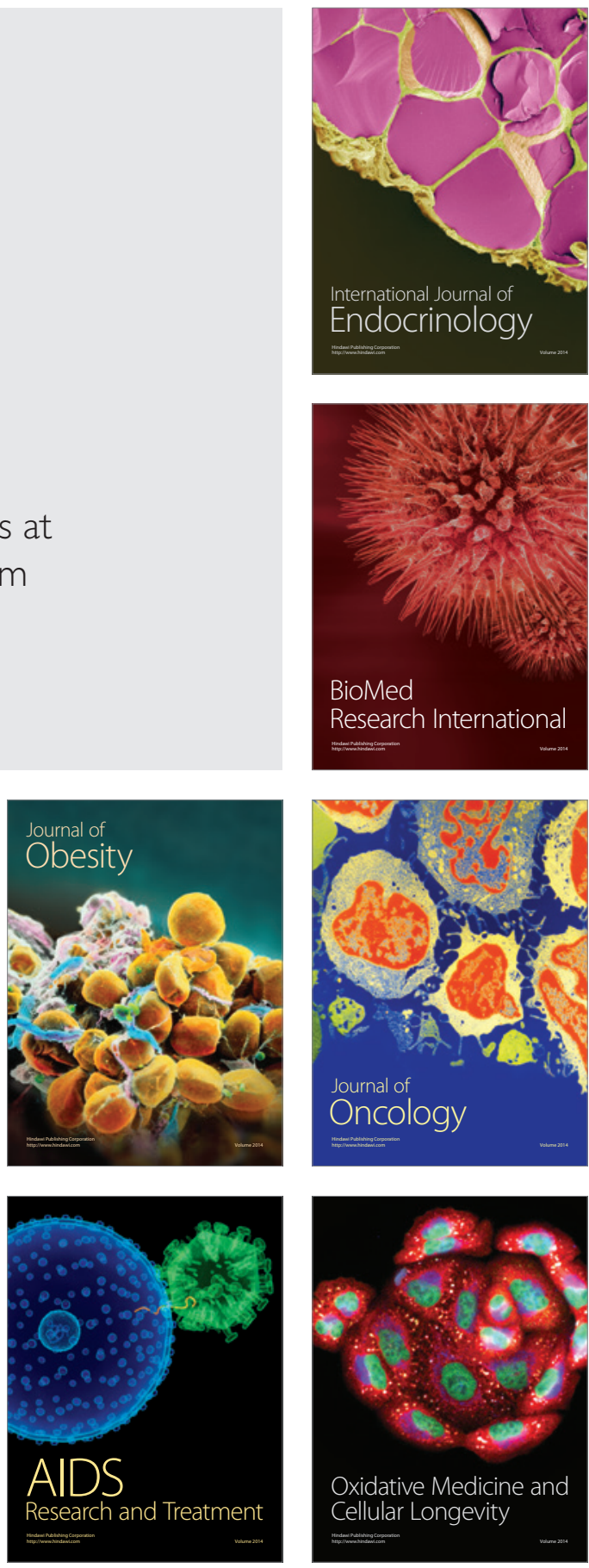